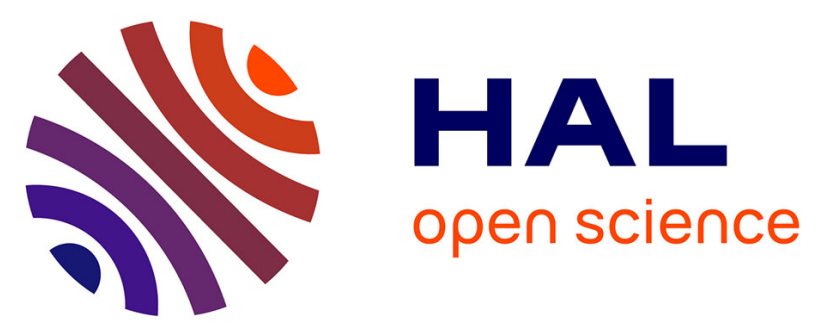

\title{
A Comparison of Two Methods for Selecting Vibrational Configuration Interaction Spaces on a Heptatomic System: Ethylene Oxyde
}

Didier Bégué, Neil Gohaud, Claude Pouchan, Patrick Cassam-Chenaï, Jacques Liévin

\section{To cite this version:}

Didier Bégué, Neil Gohaud, Claude Pouchan, Patrick Cassam-Chenaï, Jacques Liévin. A Comparison of Two Methods for Selecting Vibrational Configuration Interaction Spaces on a Heptatomic System: Ethylene Oxyde. Journal of Chemical Physics, 2007, 127, http://dx.doi.org/10.1063/1.2795711. 10.1063/1.2795711 . hal-00151806

\section{HAL Id: hal-00151806 https://hal.science/hal-00151806}

Submitted on 5 Jun 2007

HAL is a multi-disciplinary open access archive for the deposit and dissemination of scientific research documents, whether they are published or not. The documents may come from teaching and research institutions in France or abroad, or from public or private research centers.
L'archive ouverte pluridisciplinaire $\mathbf{H A L}$, est destinée au dépôt et à la diffusion de documents scientifiques de niveau recherche, publiés ou non, émanant des établissements d'enseignement et de recherche français ou étrangers, des laboratoires publics ou privés. 


\title{
A Comparison of Two Methods for Selecting Vibrational Configuration Interaction Spaces on a Heptatomic System: Ethylene Oxyde
}

\author{
Didier Bégué, Neil Gohaud and Claude Pouchan \\ Institut Pluridisciplinaire en Recherche sur l'Environnement et les Matériaux \\ (IPREM), UMR-5254-CNRS, Equipe de Chimie-Physique (ECP), IFR - rue Jules \\ Ferry - BP 27540 - F-64075 PAU Cedex France. \\ didier.begue@univ-pau.fr,claude.pouchan@univ-pau.fr \\ Patrick Cassam-Chenaï \\ Laboratoire de Mathématique J. A. Dieudonné, UMR 6621 du CNRS, Faculté des \\ Sciences, Parc Valrose, 06108 Nice cedex 2, France. cassam@unice.fr \\ Jacques Liévin \\ Université Libre de Bruxelles, Service de Chimie Quantique et Photophysique, CP \\ 160/09, 50 Av.F.D. Roosevelt, B-1050Bruxelles, Belgium.jlievin@ulb.ac.be
}

\begin{abstract}
Two recently developed methods for solving the molecular vibrational Schrödinger equation, namely, the parallel vibrational multiple window configuration interaction (P-VMWCI) and the vibrational mean field configuration interaction (VMFCI), are presented and compared on the same potential energy surface of ethylene oxide, c- $\mathrm{C}_{2} \mathrm{H}_{4} \mathrm{O}$. It is demonstrated on this heptatomic system with strong resonances that both approaches converge towards the same fundamental frequencies. This confirms their ability to tackle the vibrational problem of large molecules for which full configuration interaction calculations are not tractable.
\end{abstract}

Preprint to be published 


\section{Introduction}

The vibrational configuration interaction approach consists in diagonalizing the molecular vibrational Hamiltonian in a finite dimensional subspace of the Hilbert space associated to the system. This approximation method is known as Galerkin's method in mathematics. The variational principle insures that the larger the subspace the more accurate the results will be. However, in practice the rate of convergence depends not only on the dimension but also on the nature of the wave functions spanning the vector subspace. This dependency is crucial, as can be easily understood by considering the limit case where the variational subspace is the space spanned by the exact states searched for. Then, the diagonalisation of the Hamiltonian in this subspace will give the exact solution. Of course, this never happens in practice, however, clearly, it is advantageous to get as close as possible to this ideal case.

This issue has triggered the two variational approaches which are studied in this article, namely, the parallel vibrational multiple window configuration interaction (P-VMWCI) method $[1,2]$ and the vibrational mean-field configuration interaction (VMFCI) method [3-5]. Our goal is to assess these approaches on a molecular system out-of-reach of standard methods. We have chosen a heptatomic molecule of astrophysical and exobiological interest [6-12], ethylene oxide, $\mathrm{c}-\mathrm{C}_{2} \mathrm{H}_{4} \mathrm{O}$, see Figure 1.

Since a full CI reference calculation is not feasible to assess each method individually, it is appropriate to evaluate them one against the other. As a matter of fact, the variational space selection processes in the two CI methods are unrelated, so the two approaches can be considered as independent. Comparison with the standard vibrational self-consistent field (VSCF)/vibrational configuration interaction (VCI) approach is also provided in order to demonstrate the superiority of the new selection strategies.

The article is organized as follows: In the next section, we recall the molecular vibrational eigenproblem and the two strategies we have developed to solve it. Then, we evaluate the convergence of numerical computations on ethylene monoxide. Finally, we conclude on the reliability of the two approaches and the new insight they provide on experimental data. 


\section{Two recently developed strategies to solve the molecular vibra- tional eigenproblem}

\subsection{The molecular vibrational eigenproblem}

In this work, we consider a vibrational Hamiltonian to quartic order expressed as:

$$
H_{v i b}=H_{\text {harm }}+V_{\text {cubic }}+V_{\text {quartic }}
$$

where,

$$
\begin{aligned}
& H_{\text {harm }}=\left(\frac{\hbar}{2}\right) \sum_{s=1}^{n_{\text {vib }}} \lambda_{s}^{1 / 2}\left(p_{s}^{2}+q_{s}^{2}\right), \\
& V_{\text {cubic }}=\sum_{s, s^{\prime}, s^{\prime \prime}=1}^{n_{\text {vib }}} k_{s, s^{\prime}, s^{\prime \prime}} q_{s} q_{s^{\prime}} q_{s^{\prime \prime}}, \\
& V_{\text {quartic }}=\sum_{s, s^{\prime}, s^{\prime \prime}, s^{\prime \prime \prime}=1}^{n_{v i b}} k_{s, s^{\prime}, s^{\prime \prime}, s^{\prime \prime \prime}} q_{s} q_{s^{\prime}} q_{s^{\prime \prime}} q_{s^{\prime \prime \prime}} .
\end{aligned}
$$

In Eqs. (2) to (4), the $q_{s}$ 's are the scaled, mass-weighted, normal coordinates, the $p_{s}$ 's their conjugate momenta, with $s$ running from one to $n_{v i b}$, the number of vibrational degrees of freedom.

The harmonic Hamiltonian, $H_{\text {harm }}$ will be considered as the zero order Hamiltonian. For each degree of freedom, the wavefunctions and energies, solutions of the harmonic vibrational equation, will be denoted by $\left|n_{s}\right\rangle$ and $E_{s}^{(0)}\left(n_{s}\right)$ respectively, with,

$$
\left\langle n_{s}^{\prime} \mid n_{s}\right\rangle=\delta_{n_{s}, n_{s}^{\prime}}
$$

and

$$
E_{s}^{(0)}\left(n_{s}\right)=h c \nu_{s}\left(n_{s}+\frac{1}{2}\right)
$$

where $\nu_{s}$ is the harmonic wavenumber.

The tensor product functions $\left|n_{1}, n_{2}, \ldots, n_{n_{v i b}}\right\rangle$,

$$
\left|n_{1}, n_{2}, \ldots, n_{n_{v i b}}\right\rangle=\bigotimes_{s=1}^{n_{v i b}}\left|n_{s}\right\rangle
$$


are orthonormal and represent a base for the expansion of the vibrational wave functions $\left|\Psi_{i}\right\rangle,(i \in[1, N])$ that are searched for:

$$
\left|\Psi_{i}\right\rangle=\sum_{n_{1}, n_{2}, \ldots, n_{n_{v i b}}} C_{n_{1}, n_{2}, \ldots, n_{n_{v i b}}}^{i}\left|n_{1}, n_{2}, \ldots, n_{n_{v i b}}\right\rangle .
$$

Generally, the anharmonic vibrational Schrödinger equation:

$$
H_{v i b}|\Psi\rangle=E|\Psi\rangle
$$

is solved with the help of variational [1-4,13-26], perturbational [27-31] (although even for the one-dimensional quartic oscillator the perturbation series is strongly divergent and particularly hard to renormalize [32]), or hybrid methods [33-36]. Choosing one of these approaches is often related to the numerical precision one wants to obtain.

Ideally, the best variational approach would consist in diagonalizing the Hamiltonian $H_{v i b}$ in the space spanned by the $\left|n_{1}, n_{2}, \ldots, n_{n_{v i b}}\right\rangle$ 's. However, this becomes rapidly prohibitive as the number of degrees of freedom increases, even if a finite but realistic set of functions per degree is used. It is necessary to build variational subspaces of tractable dimensions on which the projection of the vibrational wave functions of interest is as large as possible. This issue has triggered the two variational approaches which are presented hereafter, namely, the parallel vibrational multiple window configuration interaction (P-VMWCI) method and the vibrational mean-field configuration interaction (VMFCI) method.

\section{2 $P-V M W C I$}

Performing a full VCI in a reasonable basis set for a molecule of five or more atoms is out-of-reach of present days' computer capabilities. However, the vibrational spectrum of molecules of this size becomes amenable to theoretical calculations if the total spectrum is splitted into several windows of smaller sizes.

The configuration space corresponding to a given window can be constructed iteratively, starting with an initial space, $S_{0}$, chosen by the user as a subset of the $N$-dimensional full VCI space,

$$
S=\left\{\left|n_{1}^{i}, n_{2}^{i}, \ldots n_{n_{v i b}}^{i}\right\rangle ; 1 \leq i \leq N\right\} .
$$

Let $A$ be the union of the set reduced to the identity and the set of all the excitation operators and their Hermitian conjugate (de-excitation operators) appearing with a significant coefficient in the anharmonic potential, Eqs. $(3,4)$, 
if the latter is rewritten in second quantization. That is to say, denoting by $Q_{s}^{\dagger}$, (respectively $Q_{s}$ ), the creation, (respectively annihilation), operator for mode $s$ :

$$
\begin{aligned}
& A=A_{0} \cup A_{1} \cup A_{2} \cup A_{3} \cup A_{4} \\
& A_{0}=\{1\}, \text { (no excitation) } \\
& A_{1}=\left\{Q_{s}^{\dagger}, Q_{s} \text {, such that } \exists s^{\prime},\left|k_{s, s^{\prime}, s^{\prime}}\right|>\varepsilon ; 1 \leq s, s^{\prime} \leq n_{v i b}\right\} \\
& \cup\left\{Q_{s}^{\dagger^{2}}, Q_{s}^{2} \text {, such that } \exists s^{\prime},\left|k_{s, s, s^{\prime}, s^{\prime}}\right|>\varepsilon ; 1 \leq s, s^{\prime} \leq n_{v i b}\right\} \\
& \cup\left\{Q_{s}^{\dagger^{3}}, Q_{s}^{3} \text {, such that, }\left|k_{s, s, s}\right|>\varepsilon ; 1 \leq s \leq n_{v i b}\right\} \\
& \cup\left\{Q_{s}^{\dagger^{4}}, Q_{s}^{4} \text {, such that, }\left|k_{s, s, s, s}\right|>\varepsilon ; 1 \leq s \leq n_{v i b}\right\} \text {, } \\
& A_{2}=\left\{Q_{s}^{\dagger} Q_{s^{\prime}}^{\dagger}, Q_{s}^{\dagger} Q_{s^{\prime}}, Q_{s} Q_{s^{\prime}} \text {, such that, } \exists s^{\prime \prime},\left|k_{s, s^{\prime}, s^{\prime \prime}, s^{\prime \prime}}\right|>\varepsilon ; s \neq s^{\prime} ; 1 \leq s, s^{\prime}, s^{\prime \prime} \leq n_{v i b}\right\} \\
& \cup\left\{Q_{s}^{\dagger^{2}} Q_{s^{\prime}}^{\dagger}, Q_{s}^{2} Q_{s^{\prime}}^{\dagger}, Q_{s}^{\dagger^{2}} Q_{s^{\prime}}, Q_{s}^{2} Q_{s^{\prime}} \text {, such that, }\left|k_{s, s, s^{\prime}}\right|>\varepsilon ; s \neq s^{\prime} ; 1 \leq s, s^{\prime} \leq n_{v i b}\right\} \\
& \cup\left\{Q_{s}^{\dagger^{3}} Q_{s^{\prime}}^{\dagger}, Q_{s}^{3} Q_{s^{\prime}}^{\dagger}, Q_{s}^{\dagger^{3}} Q_{s^{\prime}}, Q_{s}^{3} Q_{s^{\prime}} \text {, such that, }\left|k_{s, s, s, s^{\prime}}\right|>\varepsilon ; s \neq s^{\prime} ; 1 \leq s, s^{\prime} \leq n_{v i b}\right\} \\
& \cup\left\{Q_{s}^{\dagger^{2}} Q_{s^{\prime}}^{\dagger^{2}}, Q_{s}^{\dagger^{2}} Q_{s^{\prime}}^{2}, Q_{s}^{2} Q_{s^{\prime}}^{2} \text {, such that, }\left|k_{s, s, s^{\prime}, s^{\prime}}\right|>\varepsilon ; s \neq s^{\prime} ; 1 \leq s, s^{\prime} \leq n_{v i b}\right\} \text {, } \\
& A_{3}=\left\{Q_{s}^{\dagger} Q_{s^{\prime}}^{\dagger} Q_{s^{\prime \prime}}^{\dagger}, Q_{s} Q_{s^{\prime}}^{\dagger} Q_{s^{\prime \prime}}^{\dagger}, Q_{s} Q_{s^{\prime}} Q_{s^{\prime \prime}}^{\dagger}, Q_{s} Q_{s^{\prime}} Q_{s^{\prime \prime}},\right. \\
& \text { such that, } \left.\left|k_{s, s^{\prime}, s^{\prime \prime}}\right|>\varepsilon ; 1 \leq s, s^{\prime}, s^{\prime \prime} \leq n_{v i b} \text { all distinct }\right\} \\
& \cup\left\{Q_{s}^{\dagger} Q_{s^{\prime}}^{\dagger} Q_{s^{\prime \prime}}^{\dagger}, Q_{s}^{\dagger} Q_{s^{\prime}}^{\dagger} Q_{s^{\prime \prime}}^{2}, Q_{s}^{\dagger} Q_{s^{\prime}} Q_{s^{\prime \prime}}^{\dagger},{ }^{2}, Q_{s}^{\dagger} Q_{s^{\prime}} Q_{s^{\prime \prime}}^{2}, Q_{s} Q_{s^{\prime}} Q_{s^{\prime \prime}}^{\dagger}, Q_{s} Q_{s^{\prime}} Q_{s^{\prime \prime}}^{2},\right. \\
& \text { such that, } \left.\left|k_{s, s^{\prime}, s^{\prime \prime}, s^{\prime \prime}}\right|>\varepsilon ; 1 \leq s, s^{\prime}, s^{\prime \prime} \leq n_{v i b} \text { all distinct }\right\} \text {, } \\
& A_{4}=\left\{Q_{s}^{\dagger} Q_{s^{\prime}}^{\dagger} Q_{s^{\prime \prime}}^{\dagger} Q_{s^{\prime \prime \prime}}^{\dagger}, Q_{s} Q_{s^{\prime}}^{\dagger} Q_{s^{\prime \prime}}^{\dagger} Q_{s^{\prime \prime \prime}}^{\dagger}, Q_{s} Q_{s^{\prime}} Q_{s^{\prime \prime}}^{\dagger} Q_{s^{\prime \prime \prime}}^{\dagger}, Q_{s} Q_{s^{\prime}} Q_{s^{\prime \prime}} Q_{s^{\prime \prime \prime}}^{\dagger}, Q_{s} Q_{s^{\prime}} Q_{s^{\prime \prime}} Q_{s^{\prime \prime \prime}},\right. \\
& \text { such that, } \left.\left|k_{s, s^{\prime}, s^{\prime \prime}, s^{\prime \prime \prime}}\right|>\varepsilon ; 1 \leq s, s^{\prime}, s^{\prime \prime}, s^{\prime \prime \prime} \leq n_{\text {vib }} \text { all distinct }\right\} \text {, }
\end{aligned}
$$

where, in general, $\varepsilon=0$. However, a non negative value can be used in some cases, to reduce the cardinal of $A$.

Then, the configuration space at order $\gamma$, is defined recursively to be the space, $S_{\gamma}$, in direct interaction with $S_{\gamma-1}$. It is made of all the configurations coupled by an operator of $A$ with a configuration of $S_{\gamma-1}$ :

$$
\begin{aligned}
& S_{\gamma}=\left\{\left|n_{1}, n_{2}, \ldots, n_{n_{\text {vib }}}\right\rangle \in S, \text { such that } \exists \alpha \in \mathbb{C} ; \exists \hat{a} \in A ; \exists\left|m_{1}, m_{2}, \ldots, m_{n_{\text {vib }}}\right\rangle \in S_{\gamma-1}\right. \\
& \left.\quad\left|n_{1}, n_{2}, \ldots, n_{n_{\text {vib }}}\right\rangle=\alpha \hat{a}\left|m_{1}, m_{2}, \ldots, m_{n_{\text {vib }}}\right\rangle\right\} .
\end{aligned}
$$

Note that with this definition, we have $S_{\gamma-1} \subseteq S_{\gamma}$, and that a non negative value for $\varepsilon$ in Eqs.(11) can reduce the size of $S_{\gamma}$.

The construction of $S_{\gamma}$ as defined in Eq.(12) is simple, for the action of the operators of $A$ on HO basis functions is straightforward. This is in contrast with variation-peturbation methods [34,35], in which the iterative building of 
the configuration space is based upon perturbational criteria such as,

$$
\begin{aligned}
& S_{\gamma}=\left\{\left|n_{1}^{j}, n_{2}^{j}, \ldots, n_{n_{v i b}}^{j}\right\rangle \in S \text { such that }, \exists\left|n_{1}^{i}, n_{2}^{i}, \ldots, n_{n_{v i b}}^{i}\right\rangle \in S_{\gamma-1}\right. \\
& \left.\quad \text { and }\left|\frac{\left\langle n_{1}^{j}, n_{2}^{j}, \ldots, n_{n_{v i b}}^{j}\left|V_{\text {cubic }}\right| n_{1}^{i}, n_{2}^{i}, \ldots, n_{n_{v i b}}^{i}\right\rangle}{E_{i}-E_{j}}\right|>\varepsilon\right\}
\end{aligned}
$$

The construction of $S_{\gamma}$ as defined in Eq.(13) is time consuming because the matrix elements of every configuration in $S_{\gamma-1}$ with millions of configurations of $S$ have to be evaluated. Consequently, we reserve the use of the perturbative filter of Eq.(13) to the case where the size of the VCI matrices becomes critical, in order to eliminate weakly coupled configurations, and only when the last iterative $\gamma$-step is reached.

For each order $\gamma, p$ configuration spaces, $S_{\gamma}^{k}, 1 \leq k \leq p$, can be constructed independently, corresponding to $p$ spectral windows (as in Table 1). So, this approach lends itself in a natural way to a parallel implementation. The configuration spaces, $S_{\gamma}^{k}$, or cuts of them, can be distributed among a $z$-nodes cluster, where the $i^{\text {th }}$ node is made of $P_{i}, 1 \leq i \leq z$, processors, within a parallel SPMD (Single Program Multiple Data) scheme.

This method, that we call "parallel vibrational multiple window configuration interaction" method, generates $p$ VCI matrices, which are much smaller than the full VCI one if $\gamma$ and the $S_{0}^{k}, 1 \leq k \leq p$, are properly chosen. Moreover, for each VCI matrix (or matrix block), the use of both MPI (Message Passing Interface) and computational efforts on loops optimisation enable to distribute tasks among the processors according to their respective clock speed and workload. However, a given configuration can be involved in several windows, specially as $\gamma$ increases. This drawback is necessary to guarantee the homogeneity of the treatment of the spectral windows regardless of the initial choice, $S_{0}^{k}, 1 \leq k \leq p$. The latter, which is controled by the user with the only restriction that $\forall k,|0, \cdots, 0\rangle \in S_{0}^{k}$, makes the method particularly flexible.

The performances of the P-VMWCI algorithm are illustrated in Table 1 by the calculation of the $\nu_{C O}$ stretching frequency of the $\mathrm{H}_{2} \mathrm{CO}$ molecule investigated in a previous work [37]. This table shows how the results converge with $\gamma$, for two initial windows of different sizes; a large window covering the $0-3500 \mathrm{~cm}^{-1}$ range and a minimal-size window of $1 \mathrm{~cm}^{-1}$ around the targeted $\nu_{C O}$ frequency. The results show that the computational costs are significantly lower with the latter window, whereas the calculated wave numbers are within a few tenth of $\mathrm{cm}^{-1}$.

Note that for the sake of simplicity, we have omitted symmetry considerations in our presentation. However, in practice we make use of symmetry to deal only with symmetry-adapted matrix blocks and to reduce further the dimensions 
of the matrices to diagonalize. The same remark holds true for the method presented in the next section.

\section{$2.3 \quad V M F C I$}

Suppose that for a given partition $P$ of the vibrational degrees of freedom of an $n_{v i b}$ oscillator system, the following two conditions are met:

(1) The vibrational Hamiltonian can be expanded in a factorized form:

$$
\begin{aligned}
H_{v i b}=h_{0}+ & \sum_{j_{1}=1}^{n_{P}} h_{j_{1}}\left(I_{j_{1}}\right)+\sum_{1 \leq j_{1}<j_{2} \leq n_{P}} h_{j_{1}, j_{2}}\left(I_{j_{1}}\right) h_{j_{1}, j_{2}}\left(I_{j_{2}}\right)+ \\
& \cdots+h_{1,2, \cdots, n_{P}}\left(I_{1}\right) h_{1,2, \cdots, n_{P}}\left(I_{2}\right) \cdots h_{1,2, \cdots, n_{P}}\left(I_{n_{P}}\right),
\end{aligned}
$$

where,

$P=\left(I_{1}, I_{2}, \cdots, I_{n_{P}}\right)=\left(\left\{i_{1}^{1}, i_{2}^{1}, \cdots, i_{k_{1}}^{1}\right\},\left\{i_{1}^{2}, i_{2}^{2}, \cdots, i_{k_{2}}^{2}\right\}, \cdots,\left\{i_{1}^{n_{P}}, i_{2}^{n_{P}}, \cdots, i_{k_{n_{P}}}^{n_{P}}\right\}\right)$.

is a partition of the $n_{v i b}$ degrees of freedom into $n_{P}$ subsets $I_{1}, I_{2}, \cdots, I_{n_{P}}$, of respectively $k_{1}, k_{2}, \cdots, k_{n_{P}}$ degrees, and where $h_{j_{1}, j_{2}, \cdots, j_{k}}\left(I_{j_{l}}\right)$ denotes a vibrational operator which only depends upon operators acting on the degrees in subset $I_{j_{l}}$. Note that this condition is satisfied for all possible partitions with the Hamiltonian of Eq.(1).

(2) We have $n_{P}$ basis sets of vibrational wave functions, $\phi_{I_{j}}^{m}\left(q_{i_{1}^{j}}, \cdots, q_{i_{k_{j}}^{j}}\right)_{m \in\left\{1, d_{j}\right\}}$, with $1 \leq j \leq n_{P}$, spanning the Hilbert subspaces of dimension $d_{j}$, of physical interest for the groups of degrees of freedom in the $I_{j}$ 's.

Let $Q$ be another partition coarser than or equal to $P$, that is to say:

$$
\begin{aligned}
& Q=\left(J_{1}, J_{2}, \cdots, J_{n_{Q}}\right), \quad \text { with } n_{Q} \leq n_{P}, \\
& \text { and } \quad \forall j \in\left\{1, \cdots, n_{P}\right\}, \quad \exists k \in\left\{1, \cdots, n_{Q}\right\} \quad \text { such that, } I_{j} \subseteq J_{k} .(16)
\end{aligned}
$$

The subsets $J_{k}$ are referred to as "contractions" whose components are the $I_{j}$ 's such that $I_{j} \subseteq J_{k}$. The number of components in $J_{k}$ is denoted by $l_{k}$.

A VMFCI calculation consists in defining for each contraction, $J_{k}$, a mean field Hamiltonian obtained by averaging the vibrational Hamiltonian in the 
mean field of the other contractions $J_{l} \neq J_{k}$ and by diagonalizing it in a basis set of product functions,

$$
\Phi_{J_{k}}^{M}=\prod_{I_{j} \subseteq J_{k}} \phi_{I_{j}}^{m_{j}}\left(q_{i_{1}^{j}}, \cdots, q_{i_{k_{j}}^{j}}\right)
$$

where the superlabel $M$ stands for the sequence of indices $m_{j}$ of the component functions.

More precisely, one first builds a partial Hamiltonian by gathering all the terms of $H_{v i b}$ that involve exclusively operators acting on the $I_{j} \subseteq J_{k}$,

$$
\begin{aligned}
& H_{k}=h_{0}+\sum_{j_{1} \text { such that }, I_{j_{1}} \subseteq J_{k}} h_{j_{1}}\left(I_{j_{1}}\right)+\sum_{j_{1}<j_{2}} \sum_{\text {such that, } I_{j_{1}, I_{j_{2}} \subseteq J_{k}}} h_{j_{1}, j_{2}}\left(I_{j_{1}}\right) h_{j_{1}, j_{2}}\left(I_{j_{2}}\right)+ \\
& \cdots+\sum_{j_{1}<\cdots<j_{l_{k}} \text { such that }, I_{j_{1}}, \cdots, I_{j_{k}} \subseteq J_{k}} h_{j_{1}, \cdots, j_{l_{k}}}\left(I_{j_{1}}\right) \cdots h_{j_{1}, \cdots, j_{l_{k}}}\left(I_{j_{l_{k}}}\right) .
\end{aligned}
$$

Then, one averages $H_{v i b}-H_{k}$ over a given state, that we have chosen to be, in this work, the product of the ground states, $\prod_{I_{j} \not J_{k}} \phi_{I_{j}}^{0}\left(q_{i_{1}^{j}}, \cdots, q_{i_{k_{j}}^{j}}\right)$, of all the components of the "spectator" contractions (that is to say, not in the contraction $J_{k}$ ). We obtain the mean-field Hamiltonian :

$$
H_{k}+\left\langle\prod_{I_{j} \nsubseteq J_{k}} \phi_{I_{j}}^{0}\left(q_{i_{1}^{j}}, \cdots, q_{i_{k_{j}}^{j}}\right)\left|H_{v i b}-H_{k}\right| \prod_{I_{j} \nsubseteq J_{k}} \phi_{I_{j}}^{0}\left(q_{i_{1}^{j}}, \cdots, q_{i_{k_{j}}^{j}}\right)\right\rangle .
$$

This Hamiltonian is diagonalized in the basis set of all product functions of Eq.(17), or in a subset of the latter obtained by selecting the product functions such that the sum of the energies of their components is below a given threshold. Additionally, extra threshold can be applied to the energy of any component.

In contrast with the traditional contraction method [38-42], where the mean field term is omitted in Eq.(19), it is relevant to iterate a VMFCI calculation with the same partition, except of course in the VCI case corresponding to the partition, $\left(\left\{1,2, \ldots, n_{v i b}\right\}\right)$. This way, one can achieve a vibrational self-consistent field configuration interaction (VSCFCI) calculation. The simplest example is the VSCF case $[43,44]$ corresponding to the partition $\left(\{1\},\{2\}, \ldots,\left\{n_{v i b}\right\}\right)$, iterated until a chosen convergence criterion is met.

In summary, the VMFCI scheme is a particularly general variational method. By carrying out a basis set truncation after such a calculation, one can systematically achieve a drastic reduction of the basis set size to be used in following 
calculations, corresponding to possibly coarser partitions, without affecting the end result.

The efficiency of the mean field CI treatment is illustrated in Table 2 for the low-lying vibrational levels of methane, calculated as in our previous work [5] from the Lee, Martin, Taylor potential energy surface (PES) [45]. This table compares 3 different "minimal symmetry preserving" (MSP) calculations to a harmonic and a converged (see Table 13 of the supplementary material of [5]) calculations. Let us recall that MSP calculations actually consist in contracting degenerate components together, in order to ensure that no symmetry breaking occurs, (in contrast with VSCF calculations). For methane, such a calculation reduces the 9 modal basis sets to 4 product basis sets by contracting together the modals (i.e. vibrational one-degree of freedom functions) of the two components of the E mode and the modals of the three components of the two T2 modes, the remaining mode carries the A1 representation, so it is non degenerate. Each MSP calculation step then consists in 4 separate diagonalizations of the Hamiltonian matrix within the latter basis sets. So, their computational costs are in the same range. The MSP-CI, which corresponds to the standard contraction method without mean field treatment, only accounts for the intra-mode anharmonic coupling. It does not improve significantly the harmonic results for modes 1 and 2 and goes in the wrong direction for modes 3 and 4. Oppositely, MSP-VMFCI and MSP-VSCFCI calculations, which introduce the mean field averaging with and without iterating, provide significantly better results. MSP-VSCFCI results always go in the right direction, with modes 1 and 2 closer to the converged values than with MSP-CI. Therefore, mean field contracted wave functions provide an efficient basis set to be used in further contraction steps, which is not the case of the standard contracted wave functions. The conclusions drawn from this example have been verified in all other systems studied so far.

\section{Performances, convergence, and comparison of P-VMWCI and VMFCI approaches. An interesting test molecule: ethylene monoxyde.}

Identified in the interstellar medium, ethylene monoxyde has been the subject of numerous recent investigations [6-12]. Its IR spectrum has been studied by many authors in the gas phase $[47,48,51,52]$, the liquid phase [47], the solid phase $[49,50]$ and more recently in thin films and in amorphous water ice [12]. Assignment of some bands differs from a publication to another principally because of Fermi resonances between $A_{1}$ and $B_{1}$ modes, and because of the band vicinity in the $800-900 \mathrm{~cm}^{-1}$ area where are expected ring deformation and the $\mathrm{CH}_{2}$ rocking and twisting modes. Up to now, only harmonic or scaled harmonic force fields $[49,51,53,54]$ have been calculated at ab initio, DFT and semi-empirical levels of theory to support or validate the different experimental 
assignments. However, none of these harmonic studies allows one to dispel experimental ambiguities.

In this work, we have used an anharmonic potential energy surface (PES) which combines CCSD(T)/cc-pVTZ equilibrium geometry and harmonic constants with B3LYP $/ 6-31+\mathrm{G}(\mathrm{d}, \mathrm{p})$ cubic and quartic force constants $[55,56]$. The accurate results obtained in previous investigations on medium size systems $[55,57]$ have validated this combined approach. Furthermore, our anharmonic force field was built by taking into account energies, gradients and even hessians but only for selected distorted geometries [58,59]. All constants were deduced from our EGH code [58].

In the present study, we have mainly focused attention on the fundamental transition wave numbers. However, we have also paid attention to some specific overtones expected to show up in the region around $3000 \mathrm{~cm}^{-1}$ where the $\mathrm{CH}_{2}$ stretching vibrations are observed. An exhaustive analysis of the complete spectrum is in progress and will be published elsewhere.

The convergence of the P-VMWCI method is assessed in Table 3. This table illustrates how crucial the choice of the vibrational configuration space, (determined by its order $\gamma$ ), is for the accuracy of the results in the P-VMWCI approach. Three iterations are at least needed in the construction of the configuration space (see $\nu_{1}$ for example). This is because, on the one hand, the force constants linking the fundamental and harmonic first excited states to their overtones or to combination bands are not very strong. As a matter of fact, in our $\mathrm{C}_{2} \mathrm{H}_{4} \mathrm{O}$ anharmonic force field, it is uncommon to find constants that exceed $100 \mathrm{~cm}^{-1}$. However, on the other hand, many overtones and combination bands turn out to be quasi-degenerate, so, in the present case, it is the $\gamma=2$, indirectly interacting configurations, which bring the main contribution to the description of the fundamental transitions. The P-VMWCI results for $\gamma=3$, (see Table 5 where they are repeated), are nearly the same as the converged VMFCI ones for all the targeted vibrational levels.

Table 4 reports the results obtained with the VMFCI approach. All calculations start with a VSCF calculation, (there is no degenerate mode, so it is the MSP-VMFCI calculation), which is converged to $10^{-6} \mathrm{~cm}^{-1}$ after 7 iterations. The first column of the table reports the results obtained from a VCI contracting the 15 modes together directly after the VSCF, an energy truncation at $8400 \mathrm{~cm}^{-1}$ being applied to the sum of VSCF modal energies. The VMFCI results reported in the other columns are all obtained by applying the following 4-step contraction scheme :VSCF $/ \mathrm{VSCFCI}\left(\nu_{1}-\nu_{6}-\nu_{9}-\nu_{13}, \nu_{3}-\right.$ $\left.\nu_{5}-\nu_{12} ; 34072,17000\right) / \operatorname{VSCFCI}\left(\nu_{1}-\nu_{6}-\nu_{9}-\nu_{10}-\nu_{13}-\nu_{15} ; 19464\right) / \mathrm{VCI}(\mathrm{Z})$. The step following the VSCF calculation introduces a separate contraction of the $\mathrm{CH}$ stretching modes $\left(\nu_{1}, \nu_{6}, \nu_{9}\right.$ and $\left.\nu_{13}\right)$ and of the ring modes $\left(\nu_{3}, \nu_{5}, \nu_{12}\right)$. These contractions were suggested by preliminary calculations based on the ZPE 
lowering criterion [5]. More precisely, VMFCI calculations were performed for all possible binary (105) and ternary (456) contractions, and the results were sorted by decreasing order of the ZPE stabilization with respect to the corresponding VSCF value. The larger effects clearly occured for binary (6.2 to $6.9 \mathrm{~cm}^{-1}$ ) and ternary (12.9 to $14.9 \mathrm{~cm}^{-1}$ ) contractions involving the $\mathrm{CH}$ stretching modes, suggesting to contract in priority these modes together. The importance of the ternary contraction of the ring modes also suggested to contract these modes together. Note that at this stage, contracted modes are calculated in the mean field of the others, and that truncation energy thresholds of 34072 and $17000 \mathrm{~cm}^{-1}$ are applied to the stretching and ring mode contractions respectively. After achieving self-consistency (only one more iteration is needed), we introduce two more modes $\left(\nu_{15}\right.$ and $\left.\nu_{10}\right)$ into the $\mathrm{CH}$ stretching contraction, applying an energy cut-off of $19464 \mathrm{~cm}^{-1}$ to the resulting contraction. Again, only two VMFCI steps are needed to reach self-consistency for this contraction. Introducing the $\mathrm{CH}_{2}$ rocking mode $\nu_{15}$ is justified by the ZPE stabilization of about $9 \mathrm{~cm}^{-1}$ of ternary contractions in which $\nu_{15}$ is coupled with $\mathrm{CH}$ stretching modes. Addition of the $\mathrm{CH}_{2}$ twisting mode $\nu_{10}$ comes from the comparison of the $\mathrm{VCI}(8400)$ and a VMFCI similar to the 4-step scheme above but without contracting the $\nu_{10}$ mode and with a truncation at 7700 $\mathrm{cm}^{-1}$ in the final step, showing that an energy inversion occured between the $\nu_{1}$ and the $2 \nu_{10}$ energy levels, with an energy difference between the levels as large as $\pm 50 \mathrm{~cm}^{-1}$. A further analysis of the $\nu_{1}-\nu_{10}$ contraction showed that $\nu_{1}$ is strongly mixed with $2 \nu_{10}$ (found at $2959 \mathrm{~cm}^{-1}$ in our best calculation). Note that $\nu_{13}$ and $\nu_{15}+2 \nu_{4}\left(3045 \mathrm{~cm}^{-1}\right)$ exhibited a similar inversion but with a smaller energy splitting $\left( \pm 4 \mathrm{~cm}^{-1}\right)$ not justifying the inclusion of $\nu_{4}$ into the $\left(\nu_{1}-\nu_{6}-\nu_{9}-\nu_{10}-\nu_{13}-\nu_{15}\right)$ contraction. The fourth and last step consists in a VCI including all modes, with a global energy truncation at $\mathrm{Z} \mathrm{cm}^{-1}$. Results collected in the four last columns of Table 4 obey to the contraction scheme just described, showing the convergence with $\mathrm{Z}$ ranging from 8400 to $10721 \mathrm{~cm}^{-1}$. This scheme is clearly more effective than the direct VCI after the VSCF, as can been seen from the zero point energy (ZPE) convergence: 12501 for $\operatorname{VCI}(8400), 12464.5$ for $\operatorname{VMFCI}(8400)$, then $12464 \mathrm{~cm}^{-1}$ for the other VMFCIs (12463.7 $\mathrm{cm}^{-1}$ for VMFCI(10721) ) and by inspecting the stretching modes in Table 4 . The convergence of the fundamental wave numbers with increasing $\mathrm{Z}$ values is clear. So, the ZPE convergence also means that the excited energy levels have converged to about $1 \mathrm{~cm}^{-1}$ accuracy.

We have reported in Table 5 the best values of the P-VMWCI and VMFCI approaches. They are tabulated along with the available experimental data. The results of the two theoretical methods are very similar, the mean deviation being smaller than $0.2 \%$ i.e. $3.5 \mathrm{~cm}^{-1}$. The largest difference is found to be 6 $\mathrm{cm}^{-1}$ for the $\nu_{10}\left(\sigma_{C \mathrm{H}_{2}}\right)$ and $\nu_{15}\left(\rho_{C \mathrm{H}_{2}}\right)$ modes. 


\section{Conclusion}

The convergence of the two independent, theoretical approaches indicates their reliability. It entitles us to claim that the accuracy of our theoretical wave number values are essentially that of the CCSD(T)/B3LYP//cc-pVTZ/6$31+\mathrm{G}(\mathrm{d}, \mathrm{p})$ PES.

On the ground of our theoretical wave number values, six points can be underlined:

- Both $\nu_{6}$ and $\nu_{13} \mathrm{CH}_{2}$ asymmetric stretching modes are fairly well reproduced by our calculations (within $1 \%$ of the observed values). The gap calculated between the two modes ( 9 and $14 \mathrm{~cm}^{-1}$ ) is in perfect agreement with the observed value $\left(12 \mathrm{~cm}^{-1}\right)$ on ethylene oxyde crystals [50], if the lowest (and most intense) wave numbers are retained. However, the sign of the gap is the opposite in the most recent experiment on thin ethylene oxyde films deposited at low temperature [12] $\left(-12 \mathrm{~cm}^{-1}\right.$ for the amorphous phase and $-15 \mathrm{~cm}^{-1}$ for the crystalline phase) .

- The $\mathrm{CH}_{2}$ symmetric stretching vibrations $\nu_{1}$ and $\nu_{9}$ are calculated 110 to $130 \mathrm{~cm}^{-1}$ below the asymmetric $\nu_{6}$ and $\nu_{13}$ modes in contrast with the experimental values. A large part of this gap $\left(\approx 80\right.$ to $\left.100 \mathrm{~cm}^{-1}\right)$ is already present at the harmonic level. A Morse function fit to our polynomial PES is probably needed to remedy to these discrepancies. Moreover, several strong, coupled bands are expected in this crowded area of the spectral range. Theoretical investigations are in progress to analyse in more detail this issue.

- A perturbative treatment of the $\mathrm{CH}_{2}$ symmetric stretching vibrations not reported here, has led to 3061 and $3015 \mathrm{~cm}^{-1}$ for $A_{1}\left(\nu_{1}\right)$ and $B_{1}\left(\nu_{9}\right)$ modes respectively whereas the other wave numbers are close to the converged, variational values. These particular discrepancies of the perturbational approach have to be linked with the presence of strong interactions between cycle deformations and implying also hydrogen bending modes. Variational calculations involving all the states of the fundamental and combination bands located in the [500-10000] $\mathrm{cm}^{-1}$ area have revealed that the $\nu_{1}$ and $\nu_{9}$ fundamental bands are mainly mixed with the $\left(\nu_{5}+\nu_{7}+\nu_{8}\right.$ and $\left.\nu_{5}+\nu_{11}+\nu_{12}\right)$ $A_{1}$ overtones and the $\left(\nu_{2}+\nu_{10}, 2 \nu_{8}+\nu_{12}, \nu_{11}+2 \nu_{15}\right) B_{1}$ overtones respectively.

- All $\mathrm{CH}_{2}$ scissoring, rocking, twisting and wagging vibrations belonging to $A_{1}, B_{1}$ and $B_{2}$ symmetries, (i.e. infra-red active), are in very good agreement with experimental data.

- The ring breathing $\left(\nu_{3}\right)$ and the ring deformation $\left(\nu_{5}\right)$ respectively calculated at $1271-1272 \mathrm{~cm}^{-1}$ and $881-879 \mathrm{~cm}^{-1}$ are in perfect agreement with the 
observed bands reported at 1270 and $877 \mathrm{~cm}^{-1}$ in [49,51] for ethylene oxyde in the gas phase; The last ring deformation $\nu_{12}$ computed at $820-823 \mathrm{~cm}^{-1}$ supports Nakanaga's assignment $\left(822 \mathrm{~cm}^{-1}\right.$ [51]) against the weak absorption bands between 840 and $892 \mathrm{~cm}^{-1}$ assigned by many workers $[47,49]$ to this vibration.

- The two $\mathrm{A}_{2} \mathrm{CH}_{2}$ twisting $\left(\nu_{7}\right)$ and rocking $\left(\nu_{8}\right)$ modes inactive in the IR gas phase are calculated respectively at $\left(1149-1152 \mathrm{~cm}^{-1}\right)$ and (1019$\left.1024 \mathrm{~cm}^{-1}\right)$. This latter value in agreement with the estimated wavenumber (above about $1000 \mathrm{~cm}^{-1}$ ) from microwave spectroscopy [48] differs by about $200 \mathrm{~cm}^{-1}$ from all the other IR assignments [12,47,49-51]. In the same way, for the twisting mode $\left(\nu_{7}\right)$ the difference between our results and the other assignment is about $100 \mathrm{~cm}^{-1}$, see for example the wave number suggested by Schriver et al [12] to characterize this mode in crystalline and amorphous phases.

\section{ACKNOWLEDGEMENTS}

The Pau group acknowledges the Centre Informatique National de l'Enseignement Supérieur (CINES) for support of this work. J. L. thanks the Fond National de la Recherche Scientifique (FNRS, contracts FRFC and IISN) and the "Action de Recherches Concertées de la Communauté française de Belgique" for financial support. P. C.-C. thanks Jean-Marc Lacroix for his help in taking the best advantage of the JAD laboratory computer facilities and acknowledges the french ANR (Projet AHBE) for fundings. 


\section{References}

[1] N. Gohaud, D. Bégué, C. Darrigan, C. Pouchan, J. Comp. Chem. 26, 743-754 (2005).

[2] D. Bégué, N. Gohaud, R. Brown, C. Pouchan, J. Math. Chem. 40, 197 (2006).

[3] P. Cassam-Chenaï and J. Liévin, Int. J. Quantum Chem. 93, 245-264 (2003).

[4] P. Cassam-Chenaï, J. Quant. Spectrosc. Radiat. Transfer 82, 251-277 (2003).

[5] P. Cassam-Chenaï, J. Liévin, Journal of Computational Chemistry 27, 627-640 (2006).

[6] J.E. Dickens, W.M. Irvine, M. Ohishi, M. Ikeda, S. Ishikawa, A. Nummelin and A. Hjalmarson. Astrophys. J. 489, 753 (1997).

[7] A. Nummelin, J.E. Dickens, P. Bergman, A. Hjalmarson, W.M. Irvine, M. Ikeda and M. Ohishi. Astron. Astrophys. 337, 275 (1998).

[8] J. Pan, S. Albert, K.V.L.N. Sastry, E. Herbst, F.C. de Lucia, Astro. J. 499, 517 (1998).

[9] M. Ikeda, M. Ohishi, A. Nummelin, J. E. Dickens, P. Bergman, Å. Hjalmarson, and W. M. Irvine, Astrophys. J. 560, 792 (2001).

[10] M. Ikeda, M. Ohishi, A. Nummelin, J. E. Dickens, P. Bergman, Å. Hjalmarson, and W. M. Irvine, Astrophys. J. 571, 560 (2002).

[11] S. Chandra. Astron. Astrophys. 402, 1 (2003).

[12] A. Schriver, J.M. Coanga, L. Schriver-Mazzuoli and P. Ehrenfreund, Chem. Phys. 303, 13-25 (2004).

[13] S. Carter and J. M. Bowman, in "Rovibrational Bound States in Polyatomic Molecules", M. M. Law, I. A. Atkinson and J. M. Hutson Eds., (CCP6, Daresbury, 1999), pp. 60-67.

[14] K. M. Dunn, J. E. Boggs and P. Pulay, J. Chem. Phys 85, 5838 (1986).

[15] A. Niño and C. Muñoz-Caro, Comp. Chem. 19, 371-378 (1995).

[16] C. Muñoz-Caro and A. Niño, QCPE Program Number: 665

[17] G. M. Chaban, J. O. Jung, R. B. Gerber, J. Chem. Phys 111, 1823 (1999).

[18] F. Gatti, C. Iung, C. Leforestier and X. Chapuisat, J. Chem. Phys 111, 7236 (1999).

[19] A. Viel and C. Leforestier, J. Chem. Phys 112, 1212 (2000).

[20] J.C. Light and T. Carrington, Adv. Chem. Phys. 114, 263 (2000).

[21] N. J. Wright, R. B. Gerber, J. Chem. Phys 114, 8763 (2001). 
[22] N. Matsunaga, G. M. Chaban, R. B. Gerber, J. Chem. Phys 117, 3541 (2002).

[23] Hua-Gen Yu, J. Chem. Phys 121, 6334 (2004).

[24] Hee-Seung Lee and J. C. Light, J. Chem. Phys 120, 4626 (2004).

[25] F. Ribeiro, C. Iung, and C. Leforestier, J. Chem. Phys 123, 54106 (2005).

[26] R. Dawes and T. Carrington, J. Chem. Phys 122, 134101 (2005).

[27] L. B. Harding and W. C. Ermler, J. Comp. Chem. 6, 13 (1985).

[28] J. F. Gaw, A. Willets, W. H. Green and N. C. Handy, in Advances in molecular vibrations and collision dynamics, J. Bowman ed., (JAI Press, Greenwich, 1991).

[29] W. Thiel, Chimia Int. J. Chem. 58, 276 (2004).

[30] V. Barone, G. Festa, A. Grandi, N. Rega and N. Sanna, Chem. Phys. Lett. 388, 279-283 (2004).

[31] V. Barone, J. Chem. Phys 122, 14108 (2005).

[32] E. J. Weniger, Annals of Phys. 246, 133 (1996).

[33] S. Brodersen and J.-E. Lolck, J. Mol. Spectrosc. 126, 405-426 (1987).

[34] C. Pouchan and K. Zaki, J. Chem. Phys 107, 342 (1997).

[35] I. Baraille, C. Larrieu, A. Dargelos, M. Chaillet, Chem. Phys. 273, 91 (2001).

[36] O. Christiansen, J. Chem. Phys 120, 2149 (2004).

[37] D. Bégué, S. Elissalde, E. Péré, P. Iratçabal, C. Pouchan, J. Phys. Chem. A110, 7793 (2006).

[38] B. Maessen and M. Wolfsberg, J. Phys. 89, 3876 (1985).

[39] C. L. Chen, B. Maessen, and M. Wolfsberg, J. Chem. Phys 83, 1795 (1985).

[40] J. Tennyson, Comp. Phys. Rep. 4, 1 (1986).

[41] S. Carter and N. C. Handy, Comp. Phys. Rep. 5, 15 (1986).

[42] Z. Bačić and J. C. Light, Annu. Rev. Phys. Chem. 40, 469 (1989).

[43] G. D. Carney, L. L. Sprandel and C. W. Kern in "Advances in Chemical Physics", Vol. 37, I. Prigogine and S.A. Rice, Eds., (Wiley, New York, 1978), pp. 305-379.

[44] R. B. Gerber and M. A. Ratner, Chem. Phys. Lett. 68, 195 (1979).

[45] T. J. Lee, J. M. L. Martin and P. R. Taylor, J. Chem. Phys 102, 254 (1995).

[46] R. Burcl, S. Carter, N. C. Handy, Chem. Phys. Lett. 373, 357-365 (2003).

[47] R.C. Lord, B. Nolin, J. Chem. Phys 24, 656 (1956). And refs. therein to earlier works. 
[48] N. Yoshimizu, C. Hirose, S. Maeda, Bull. Chem. Soc. Jap. 48, 3529 (1975).

[49] N.W. Cant, W.J. Armstead, SpectroChimica Acta A31, 839 (1975).

[50] J.E. Bertie, S.M. Jacobs, J. Chem. Phys 68, 97 (1978).

[51] T. Nakanaga, J. Chem. Phys 73, 5451 (1980). And refs. therein to earlier works.

[52] T. Nakanaga, J. Chem. Phys 74, 5384 (1981).

[53] M.A. Lowe, J.S. Alper, R. Kawiecki, P.J. Stephens, J. Phys. Chem. 90, 41 (1986).

[54] K. Frimand, K.J. Jalkanen, Chem. Phys. 279, 161 (2002).

[55] D. Bégué, Ph. Carbonnière, C. Pouchan, J. Phys. Chem. A109, 4611 (2005).

[56] A.D. Boese, W. Klopper, J.M.L. Martin, Int. J. Quantum Chem. 104, 830 (2005).

[57] P. Carbonniere, T. Lucca, C. Pouchan, N. Rega, V. Barone, J. Comp. Chem. 384, 26 (2005).

[58] P. Carbonnière, D. Bégué, A. Dargelos, C. Pouchan, Chem. Phys. 300, 41 (2004).

[59] R. Dawes, D.L. Thompson, Y. Guo, A.F. Wagner, M. Minkoff, J. Chem. Phys 126, 184108 (2007). 


\section{List of Figure captions}

Figure 1: Schematic representation of ethylene oxyde. 


\begin{tabular}{c|c|c|c||c|c|c|} 
& \multicolumn{5}{|c||}{$[3500]$} & \multicolumn{3}{|c|}{$[1]$} \\
\hline$\gamma$ & $S_{\gamma}{ }^{\mathrm{a}}$ & $N_{o p}{ }^{\mathrm{b}}$ & $\nu_{C O}$ & $S_{\gamma}{ }^{\mathrm{a}}$ & $N_{o p} \mathrm{~b}$ & $\nu_{C O}$ \\
0 & $18(0.1)$ & - & 1781.2 & 2 & - & 1781.2 \\
1 & $976(3.3)$ & 7913 & 1754.8 & $336(1.1)$ & 768 & 1755.2 \\
2 & $6610(22.3)$ & 554004 & 1750.8 & $3449(11.6)$ & 180376 & 1750.9 \\
3 & $17783(59.9)$ & 3981840 & 1750.2 & $12526(42.2)$ & 2062647 & 1750.4 \\
4 & $26737(90.1)$ & 10608135 & 1750.0 & $23329(78.6)$ & $7550757^{\mathrm{c}}$ & 1750.2 \\
\hline
\end{tabular}

Table 1

Comparison of P-VMWCI approach performances considering two spectral windows with a decreasing density of states : Illustration with the $\nu_{C O}$ stretching mode of the $\mathrm{H}_{2} \mathrm{CO}$ molecule, obtained from a potential function computed at the CCSD(T)/ccpVQZ level of theory [37]. The [3500] window, which covers the $0-3500 \mathrm{~cm}^{-1}$ spectral area, is initially composed of 18 configurations. The second window, namely the [1] window, is $1 \mathrm{~cm}^{-1}$ wide around the perturbational value of the $\nu_{C O}$ wave number and only contains the $\nu_{C O}$ wave function (plus the ground state). Considering a reduced-sized window enables to have $25 \%$ operations less. This also reduces the problem size by $12 \%$ and CPU needs by $50 \%$. Accuracy for the $\nu_{C O}$ mode is equal to $0.17 \mathrm{~cm}^{-1}$ when $\gamma=4$.

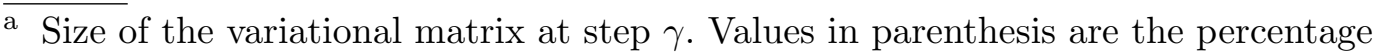
of configurations contained in $S_{\gamma}$ with respect to the total number $N=29680$ of configurations; $N$ corresponds to 7 excitation quanta for the $\tau_{C H_{2}}$ and $\nu_{C H_{2}}$ modes, and 6 excitation quanta for the $\rho_{C H_{2}} \sigma_{C H_{2}}, \nu_{C O}$ and $\nu_{a S_{C H_{2}}}$ modes. The P-VMWCI automatically rejects configurations which do not meet theses criteria.

b The $N_{o p}$ column displays the number of operations required by the action of the operators in $A$ to build the configuration space of step $\gamma$. 


\begin{tabular}{c|ccccc|} 
& Harmonic level & MSP-CI & MSP-VMFCI & MSP-VSCFCI & Converged \\
\hline$\nu_{4}$ & 1345 & $\mathbf{1 3 5 6}$ & 1295 & 1318 & 1309 \\
$\nu_{2}$ & 1570 & 1567 & 1527 & 1532 & 1528 \\
$2 \nu_{4}$ & 2691 & $\mathbf{2 7 1 4}$ & 2591 & 2638 & 2588 \\
$2 \nu_{4}$ & 2691 & $\mathbf{2 7 1 9}$ & 2597 & 2643 & 2610 \\
$2 \nu_{4}$ & 2691 & $\mathbf{2 7 1 9}$ & 2597 & 2643 & 2622 \\
$\nu_{1}$ & 3036 & 3013 & 2972 & 2972 & 2925 \\
$\nu_{3}$ & 3157 & $\mathbf{3 2 1 4}$ & $\mathbf{3 1 7 6}$ & 3062 & 3027 \\
$2 \nu_{2}$ & 3141 & 3131 & 3051 & 3061 & 3051 \\
$2 \nu_{2}$ & 3141 & 3134 & 3054 & 3064 & 3054 \\
$3 \nu_{4}$ & 4036 & $\mathbf{4 0 7 9}$ & 3896 & 3966 & 3868 \\
$3 \nu_{4}$ & 4036 & $\mathbf{4 0 8 7}$ & 3905 & 3974 & 3905 \\
$3 \nu_{4}$ & 4036 & $\mathbf{4 0 8 8}$ & 3905 & 3974 & 3915 \\
$3 \nu_{4}$ & 4036 & $\mathbf{4 0 8 8}$ & 3906 & 3974 & 3929 \\
$3 \nu_{2}$ & 4711 & 4694 & 4573 & 4588 & 4573 \\
$3 \nu_{2}$ & 4711 & 4699 & 4579 & 4594 & 4579 \\
$3 \nu_{2}$ & 4711 & 4699 & 4579 & 4594 & 4579 \\
\hline
\end{tabular}

Table 2

Contraction methods with and without mean field averaging. The wave numbers in $\mathrm{cm}^{-1}$ of the vibrational levels of methane are tabulated for different minimal symmetry preserving (MSP) methods (i.e. methods which contract degenerate components together, otherwise the symmetry is broken [46]). MSP-CI, which correspond to the traditional contraction method goes in the wrong direction for modes 3 and 4, that is in half of the cases. MSP-VSCFCI always go in the right direction and modes 1 and 2 are closer to the converged value than with MSP-CI. (HO modal basis set with quantum number less than in 10 all MSP calculations. The Lee, Martin, Taylor PES was used [45]). 


\begin{tabular}{c|c|c|c|c|}
\multirow{2}{*}{$A_{1}$} & \multicolumn{3}{|c|}{ P-VMWCI } \\
& Assignment & $\gamma=1$ & $\gamma=2$ & $\gamma=3$ \\
\hline & $\nu_{1}$ & 3056 & 2977 & 2922 \\
& $\nu_{2}$ & 1502 & 1501 & 1499 \\
& $\nu_{3}$ & 1272 & 1272 & 1271 \\
& $\nu_{4}$ & 1136 & 1136 & 1120 \\
& $\nu_{5}$ & 882 & 881 & 881 \\
\hline \multirow{2}{*}{$A_{2}$} & $\nu_{6}$ & 3042 & 3032 & 3027 \\
& $\nu_{7}$ & 1157 & 1154 & 1152 \\
& $\nu_{8}$ & 1026 & 1025 & 1024 \\
\hline$B_{1}$ & $\nu_{9}$ & 3012 & 2912 & 2908 \\
& $\nu_{10}$ & 1474 & 1474 & 1474 \\
& $\nu_{11}$ & 1131 & 1131 & 1130 \\
& $\nu_{12}$ & 824 & 822 & 820 \\
\hline$B_{2}$ & $\nu_{13}$ & 3057 & 3046 & 3041 \\
& $\nu_{14}$ & 1152 & 1151 & 1151 \\
& $\nu_{15}$ & 802 & 801 & 800 \\
\hline \hline
\end{tabular}

Table 3

Convergence of the P-VMWCI scheme. Fifteen minimal $\left( \pm 1 \mathrm{~cm}^{-1}\right.$ around each selected wave number) initial windows are used for the calculation of the fundamental wave numbers. Every tensor product of $\mathrm{HO}$ modals up to $n_{s}=7$ for each mode $s$ has been included in the iterative selection process. At order $\gamma=3$, the VCI matrices have a size of about $50000 \times 50000$. They have been diagonalized by a recently implemented block-Davidson algorithm. 


\begin{tabular}{l|l|l|l|l|l|l|} 
& Assignment & $\begin{array}{l}\text { VSCF/VCI(8400) } \\
(42113 \mathrm{bf})\end{array}$ & $\begin{array}{l}\text { VMFCI(8400) } \\
(46525 \mathrm{bf})\end{array}$ & $\begin{array}{l}\text { VMFCI(9200) } \\
(91052 \mathrm{bf})\end{array}$ & $\begin{array}{l}\text { VMFCI(10000) } \\
(172204 \mathrm{bf})\end{array}$ & $\begin{array}{l}\text { VMFCI(10721) } \\
(298194 \mathrm{bf})\end{array}$ \\
\hline$A_{1}$ & $\nu_{1}{ }^{\mathrm{a}}$ & 2994 & 2927 & 2921 & 2920 & 2919 \\
& $\nu_{2}$ & 1501 & 1500 & 1500 & 1497 & 1497 \\
& $\nu_{3}$ & 1273 & 1274 & 1273 & 1271 & 1272 \\
& $\nu_{4}$ & 1128 & 1128 & 1126 & 1123 & 1123 \\
\hline \multirow{2}{*}{$A_{2}$} & $\nu_{5}$ & 881 & 881 & 879 & 879 & 879 \\
& $\nu_{6}$ & 3093 & 3040 & 3034 & 3033 & 3032 \\
& $\nu_{7}$ & 1153 & 1153 & 1151 & 1149 & 1149 \\
\hline$B_{1}$ & $\nu_{8}$ & 1024 & 1024 & 1020 & 1019 & 1019 \\
& $\nu_{9}$ & 3040 & 2922 & 2915 & 2914 & 2913 \\
& $\nu_{10}$ & 1473 & 1471 & 1471 & 1468 & 1468 \\
& $\nu_{11}$ & 1130 & 1130 & 1128 & 1125 & 1125 \\
& $\nu_{12}$ & 825 & 825 & 823 & 823 & 823 \\
\hline$B_{2}$ & $\nu_{13}{ }^{\mathrm{a}}$ & 3105 & 3052 & 3044 & 3042 & 3041 \\
& $\nu_{14}$ & 1151 & 1151 & 1149 & 1147 & 1147 \\
& $\nu_{15}$ & 801 & 796 & 794 & 793 & 794 \\
\hline
\end{tabular}

Table 4

Convergence of the VMFCI scheme with the final step truncation threshold: the $\operatorname{VMFCI}(Z)$ scheme used in this study is: $\operatorname{VSCF} / \operatorname{VSCFCI}\left(\nu_{1}-\nu_{6}-\nu_{9}-\nu_{13}, \nu_{3}-\nu_{5}-\nu_{12} ; 34072\right.$, $17000) / \operatorname{VSCFCI}\left(\nu_{1}-\nu_{6}-\nu_{7}-\nu_{9}-\nu_{10}-\nu_{13} ; 19464\right) / \mathrm{VCI}(\mathrm{Z})$, that is to say, after a VSCF, the $\mathrm{CH}$ stretching modes, $\nu_{1}, \nu_{6}, \nu_{9}, \nu_{13}$, have been contracted together in the mean field of the other modes with a truncation threshold on the sum of the energies of product basis function components equal to $34072 \mathrm{~cm}^{-1}$, at the same step the ring modes, $\nu_{3}, \nu_{5}, \nu_{12}$, have been contracted together with a truncation threshold of $17000 \mathrm{~cm}^{-1}$; after achieving self-consistency (only one more iteration was needed) the $\mathrm{CH}$ stretching modes have been contracted with $\nu_{7}\left(\mathrm{CH}_{2}\right.$ twist $)$ and $\nu_{10}\left(\mathrm{CH}_{2}\right.$ scissor) with truncation of the basis set at $19464 \mathrm{~cm}^{-1}$ (self-consistency achieved in two steps); finally, all the modes were contracted with truncation at $\mathrm{Z} \mathrm{cm}^{-1}$. This scheme proved more effective than the direct VCI after the VSCF.

\footnotetext{
a In the VMFCI calculations $\nu_{1}$ was strongly mixed with $2 \nu_{10}\left(2959 \mathrm{~cm}^{-1}\right)$, and $\nu_{13}$ with $\nu_{15}+2 \nu_{4}(3045$ $\mathrm{cm}^{-1}$ ), so that, our assignment is only indicative.
} 


\begin{tabular}{|c|c|c|c|c|c|c|c|c|c|c|}
\hline \multirow{3}{*}{\multicolumn{2}{|c|}{ Assignment }} & \multirow{2}{*}{\multicolumn{2}{|c|}{$\begin{array}{c}\text { Gaz phase } \\
\text { IR }\end{array}$}} & \multirow{3}{*}{$\begin{array}{c}\text { Liquid phase } \\
\text { Raman } \\
{[47]}\end{array}$} & \multicolumn{3}{|c|}{ Solid Phase } & \multirow{2}{*}{\multicolumn{2}{|c|}{ Computed }} & \multirow{3}{*}{ type of motion } \\
\hline & & & & & \multirow{2}{*}{$\begin{array}{c}\text { amorphous } \\
{[12]}\end{array}$} & \multicolumn{2}{|c|}{ crystalline } & & & \\
\hline & & {$[51]$} & {$[49]$} & & & {$[50]$} & {$[12]$} & P-VMWCI & VMFCI & \\
\hline \multirow{5}{*}{$A_{1}$} & $\nu_{1}$ & - & 3018 & 3005 & 3011 & 3024 & 3005 & 2922 & 2919 & $\nu_{s, C H_{2}}$ \\
\hline & $\nu_{2}$ & 1497 & 1498 & 1490 & 1492 & $1481-1495$ & - & 1499 & 1497 & $\sigma_{\mathrm{CH}_{2}}$ \\
\hline & $\nu_{3}$ & 1270 & 1270 & 1266 & 1267 & $1266-1268$ & 1266 & 1271 & 1272 & Ring breath. \\
\hline & $\nu_{4}$ & - & 1148 & 1120 & - & $1119-1129$ & 1147 & 1120 & 1123 & $\omega_{\mathrm{CH}_{2}}$ \\
\hline & $\nu_{5}$ & 877 & 877 & 867 & 856 & $857-875$ & 854 & 881 & 879 & Ring deform. \\
\hline \multirow{3}{*}{$A_{2}$} & $\nu_{6}$ & - & ia & 3063 & 3073 & $3050-3051$ & 3073 & 3027 & 3032 & $\nu_{a s, C H_{2}}$ \\
\hline & $\nu_{7}$ & ia & ia & $(1345)$ & 1043 & $1043-1046$ & 1046 & 1152 & 1149 & $\tau_{\mathrm{CH}_{2}}$ \\
\hline & $\nu_{8}$ & $1020^{a}$ & ia & 807 & 821 & $837-851$ & 818 & 1024 & 1019 & $\rho_{\mathrm{CH}_{2}}$ \\
\hline \multirow{4}{*}{$B_{1}$} & $\nu_{9}$ & - & 3006 & 3005 & 3001 & 2996-3006 & 2995 & 2908 & 2913 & $\nu_{s, C H_{2}}$ \\
\hline & $\nu_{10}$ & 1470 & 1472 & - & 1467 & $1455-1467$ & 1467 & 1474 & 1468 & $\sigma_{\mathrm{CH}_{2}}$ \\
\hline & $\nu_{11}$ & - & 1151 & 1150 & 1169 & 1159-1169 & 1152 & 1130 & 1125 & $\omega_{\mathrm{CH}_{2}}$ \\
\hline & $\nu_{12}$ & 822 & 840 & 867 & - & $816-825$ & 859 & 820 & 823 & Ring deform. \\
\hline \multirow{3}{*}{$B_{2}$} & $\nu_{13}$ & 3065 & 3065 & 3063 & 3061 & $3062-3075$ & 3058 & 3041 & 3041 & $\nu_{a s, C H_{2}}$ \\
\hline & $\nu_{14}$ & - & 1142 & 1150 & - & $1146-1161$ & 1160 & 1151 & 1147 & $\tau_{\mathrm{CH}_{2}}$ \\
\hline & $\nu_{15}$ & 808 & 821 & - & 794 & 794-798 & 796 & 800 & 794 & $\rho_{\mathrm{CH}_{2}}$ \\
\hline
\end{tabular}

Table 5

Experimental and calculated wavenumbers (in $\mathrm{cm}^{-1}$ ) for the ethylene oxide compound. ia: inactive, $\nu_{s}$ : symmetrical stretching, $\nu_{a s}$ : asymmetrical stretching, $\rho$ : rocking, $\sigma$ : scissoring, $\tau$ : twisting, $\omega$ : wagging, breath.: breathing, deform.: deformation.

a From ref.[48] according to [51]. Actually Yoshimizu et al. [48] assigned this wave number to $\nu_{7}$, however their results support a value for $\nu_{8}$ above about $1000 \mathrm{~cm}^{-1}$ 


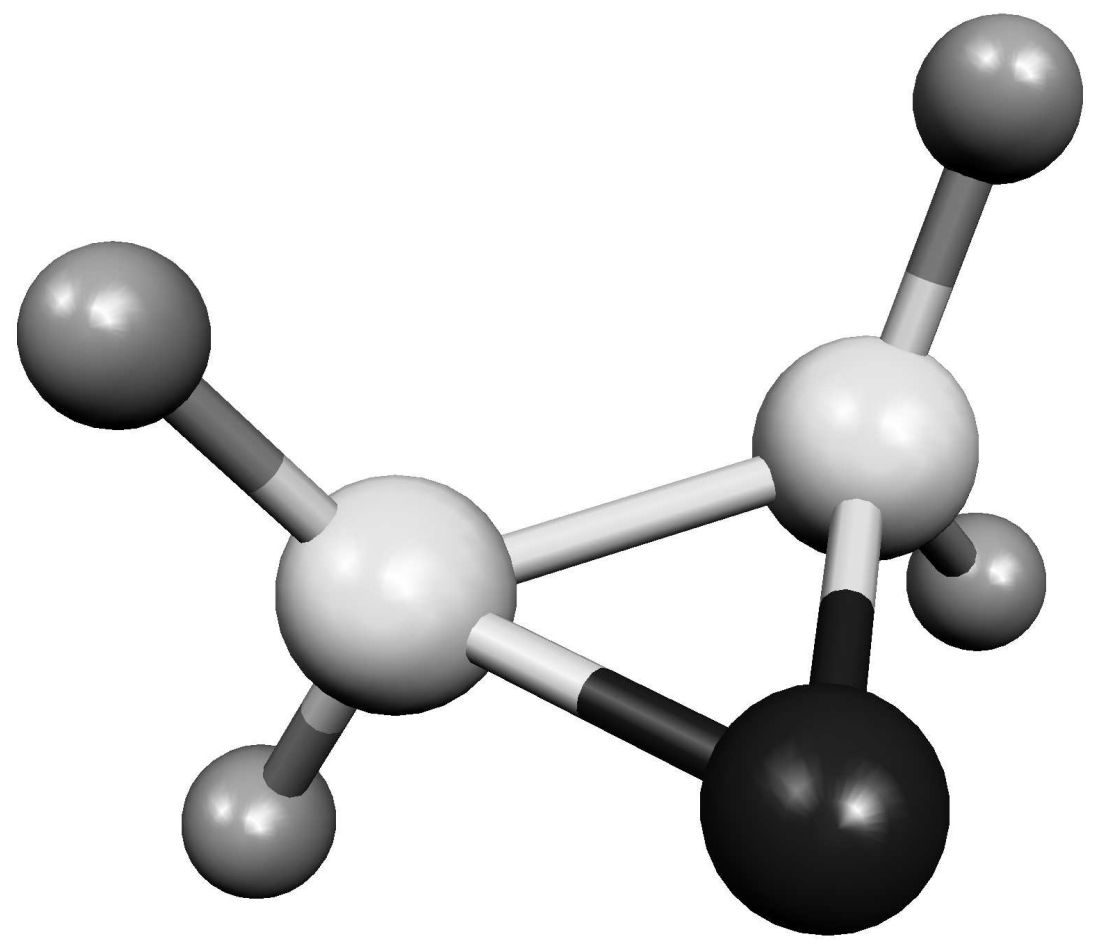

Figure 1. Bégué, Journal of Chemical Physics 